\title{
Analysis of dispersion properties of elliptic air hole photonic crystal fiber
}

\author{
Yuh-Sien Sun ${ }^{1}$, Yuan-Fong Chau ${ }^{1}$, Din-Ping Tsai ${ }^{2}$ \\ ${ }^{1}$ Department of Electronic Engineering, Chin Yun University, Jung Li, Taiwan, R.O.C. \\ ${ }^{2}$ Department of Physics, National Taiwan University, Taipei Taiwan, R.O.C.
}

\begin{abstract}
The two dimensional with a triangular-lattice cross sectional pattern of elliptic air holes photonic crystal fiber (PCF) is investigated in detail by use of plan wave expansion (PWE) method. Using PWE method, the dependence of dispersion on structure parameters is calculated. We compared the dispersion relations between the circular air holes PCF and the elliptic one. We find that the flatter dispersion curve and single mode in PCF can be realized by adjusting the width and the holes pitch. The single mode can be obtained by rotating the angle of elliptic air hole and two modes will become one mode. As an example, how to design the structure parameters for engineering the chromatic dispersion of elliptic air holes PCF is demonstrated.
\end{abstract}

Keywords: photonic crystal fiber (PCF), Plan wave expansion (PWE), elliptic air hole PCF

\section{Introduction}

Photonic crystal fibers (PCFs) are made from single material such as silica glass $\left(\mathrm{SiO}_{2}\right)$, with an array of microscopic air channels running along its length [1-7]. A defect can be created by filling the central air-hole with glass to guide light by total internal reflection (TIR) between the solid core and the cladding region with multiple air-holes $[1,2,5]$. The index-guiding PCFs are also called TIR-PCFs. Another PCFs use a perfectly period structure exhibiting a photonic band-gap (PBG) effect $[3,4,6]$ at the operating wavelength to guide light in a low index core-region. PCFs are attracting much attention in recent years because of their unique properties that are not realized in conventional optical fibers.

The desirable property of PCFs is that, the additional design parameters of air hole diameter $\mathrm{d}$, and holes pitch $\Lambda$, offer much greater flexibility in the design of dispersion to get the required application. By manipulating circular air-hole diameter $\mathrm{d}$ and pitch $\Lambda$, it is possible to control the PCF dispersion properties, for example, to change the zero-dispersion wavelength or to engineer the dispersion curve to be ultra-flattened. Furthermore, the circular air holes can be replaced by the elliptic ones, in order to obtain more flattened dispersion curve [7].

In this paper, we focus on the change of dispersion curve by replacing the circular air-holes with elliptic air-holes, as shown in Fig. 1. The elliptic air holes are described with width a and height $b$. This controlling technique is applied to design PCF with ultra-low and ultra-flattened dispersion properties. Plan wave expansion (PWE) method [8] is used to analyze the dispersion properties. Numerical results have shown that dispersion curve varied with the ratio of a and $\Lambda$, as discussed in section 2. High index core triangular elliptic air hole PCF with the zerodispersion wavelength over a wide range of wavelength has been thus designed.

\section{Results and Discussion}

The PCF sample (made by our research group) discussed here is constructed of silica ( $\mathrm{SiO} 2)$ with refractive index of $\mathrm{n}=1.492$ and its SEM cross sectional image is shown in Fig. 1.

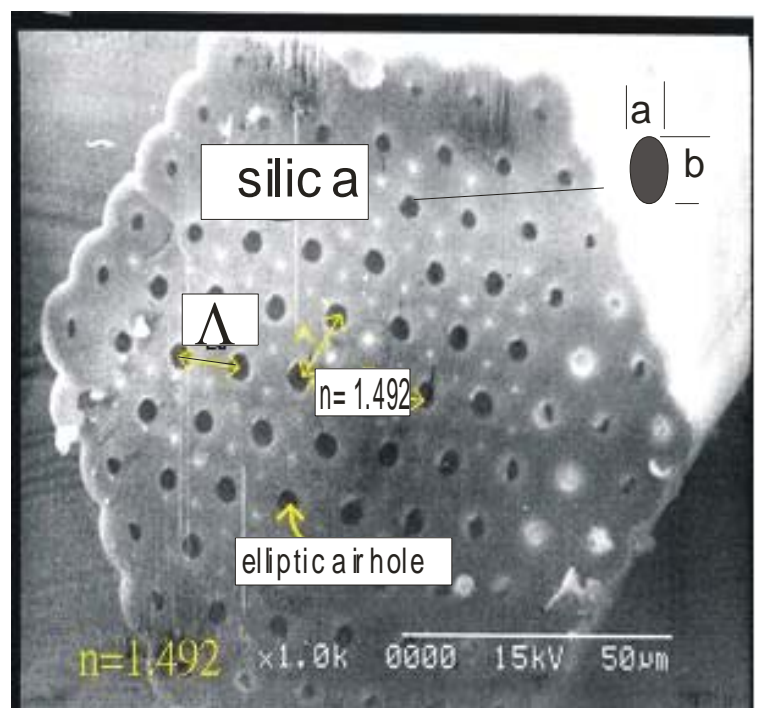


Fig. 1:The SEM cross sectional image of elliptic air hole photonic crystal with triangular lattice.

As depicted in Fig. 1, a triangular PCF with elliptic air holes is design with holes pitch $\Lambda$, width of ellipse a, height of ellipse $b$. In this section we will address the modified dispersion curve when PCFs' structure parameters are altered. PWE method is highly suited for the analysis of the triangular PCF and is one of the major tools for the understanding of PCFs [1,3]. The effective index of the fundamental space filling mode (FSM), i. e., the propagation constant of the cladding area, is given by $\mathrm{n}_{\mathrm{eff}}=\beta_{\mathrm{FSM}} / \mathrm{k}_{0}$, where $\beta_{\mathrm{FSM}}$ is the propagation constant of the FSM, $\mathrm{k}_{0}=2 \pi / \lambda$ is the free space wave number. Once the modal effect indexes $n_{\text {eff }}$ is solved, the dispersion parameter (waveguide dispersion) can be obtained. Basically, the waveguide dispersion is strongly related to the design parameters of the PCFs and therefore can be optimized to achieve desired dispersion properties. Fig. 2 shows the relation between effective index and frequency of circular air holes $(\mathrm{a}=\mathrm{b}$ case, dashed line $)$ and elliptic air hole $(\mathrm{a} \neq \mathrm{b}$ case, solid line), respectively. The geometric characteristics of the PCF are the pitch $\Lambda=2.3 \mu \mathrm{m}$, $\mathrm{a}=0.3 \Lambda$ and $\mathrm{a} / \mathrm{b}=0.83$ (for elliptic one). It shows that the effective index curve flattens with the increase of frequency and finds that the elliptic air hole reaches steady state at lower frequency. It can be evidence that elliptic air hole PCF has a better dispersion characteristic than the circular one.

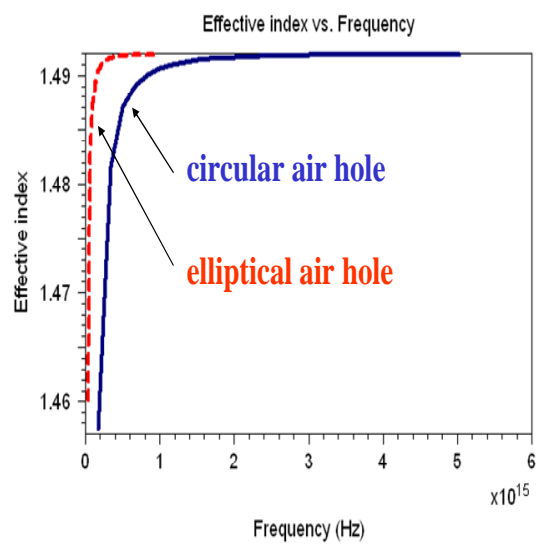

Fig. 2: Effective index as a function of frequency between circular air hole and elliptic air hole.

As expected for fiber modes, the longitudinal zcomponents are much weaker. We find that the elliptic air hole PCF has two bound modes (Fig. 3(a) and (b)) and one unbounded mode (Fig. 3(c)) in the region of elliptic air hole PCF as shown in Fig. 3. In order to obtain one single mode in PCF, the elliptic air hole is rotated by an angle of $15^{\circ}$ as shown in Fig. 4(a). It can be seen in Fig. 4(b) that a simple bounded mode is displayed in the core region of the rotated elliptic air hole PCF.

(a)

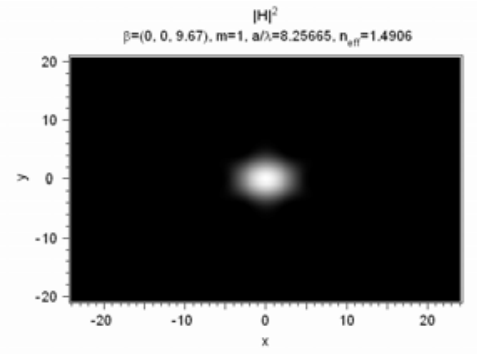

(b)

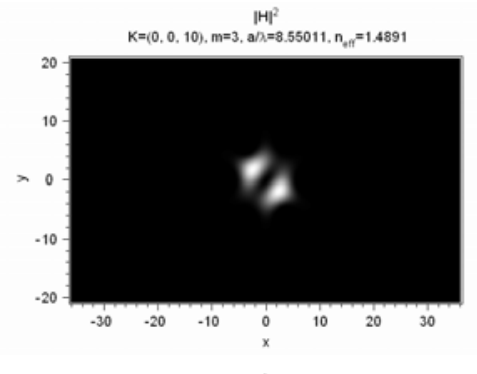

(c)

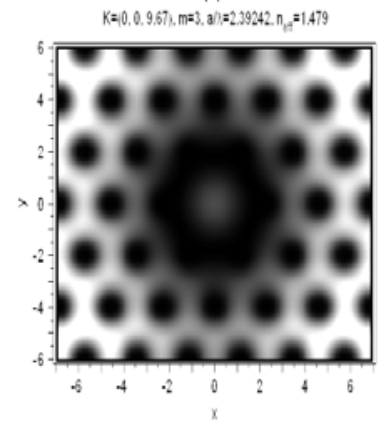

Fig. 3: Distribution of $H_{x}$ in fundamental mode of elliptic air hole PCF, where $a / b=0.83$, where (a) and (b) are two bounded mode, (c) is a unbounded mode.

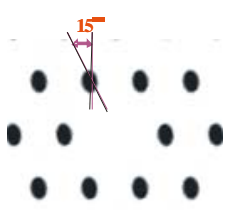

(a)

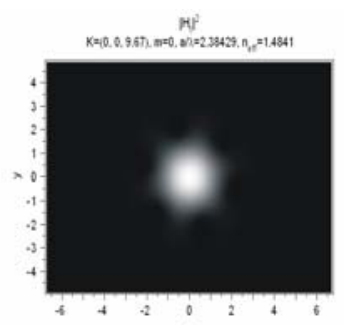

(b)
Fig. 4: Distribution of $\mathrm{H}_{\mathrm{x}}$ in fundamental mode of elliptic air hole photonic crystal, where $\mathrm{a} / \mathrm{b}=0.83$

In a conventional fiber, the number of bound modes is governed by the $\mathrm{V}$ number, which increases without limit as the wavelength decreases. It is possible to define an effective V number for PCF that 
indicates reasonably accurately whether or not a fiber is single-mode. The definition is

$V_{\text {eff }}=2 \pi \frac{\Lambda}{\lambda} \sqrt{n_{0}^{2}-n_{\text {eff }}^{2}}$

where $\Lambda$ is the air holes pitch, $\mathrm{n}_{0}$ is the silica index and $n_{\text {eff }}$ is an effective cladding index. The effective cladding index can be considered as the effective index of the first radiation state, which is equivalent to finding the lowest mode in the band structure of the plain lattice. It is shown in Ref. [1] that in contrast to step-index fibers, the $\mathrm{V}_{\text {eff }}$ for a PCF converges to a finite value as the wavelength decreases. If the finite value is less than approximately 2.405 , the PCF is single-mode. The stationary $\mathrm{V}_{\text {eff }}$ number is defined by the ratio of the hole diameter to the hole pitch $\Lambda$, and increases with the ratio. Thus by designing an elliptic air hole PCFs with $\mathrm{a} / \Lambda$ below a certain value, $V_{\text {eff }}$ number may be kept under the second-order mode cutoff value over any wavelength range, thereby ensuring an endlessly single-mode operation. For a conventional step-index fibers this cutoff $\mathrm{V}$ number is 2.405 , and although a different value is expected for the PCFs, a cutoff $\mathrm{V}_{\text {eff }}$ can be approximately 2.5 has been experimentally obtained [9].

We will evaluate $V_{\text {eff }}$ for a range of wavelengths and hole sizes. To find $n_{\text {eff }}$, we only need to simulate one unit cell of the elliptic fiber lattice, and solve for the zeroth eigenvalue. In our case, we change the value of the width of ellipse a, while all the other geometric characteristics of PCF, that is the pitch $\Lambda$, height of the ellipse $b$ and the rotational angle of elliptic air hole $\theta$, are kept constant at $\Lambda=2.3 \mu \mathrm{m}, \mathrm{b}=0.5727 \mu \mathrm{m}$ and $\theta=15^{0}$. Fig. 5 shows the variation of $V_{\text {eff }}$ with $\log _{10}(\Lambda / \lambda)$ for various relative ratio $\mathrm{a} / \Lambda$. The horizontal line corresponds to the single mode condition, $\mathrm{V}_{\text {eff }}=2.405$, the cutoff $\mathrm{V}$ value for a step-index fiber. The $\lambda \rightarrow 0$ limit of Veff approaches zero slowly as a/ $\lambda$ approaches zero.

The fiber of our sample as shown in Fig. 1 has $\Lambda=2.3 \mu \mathrm{m}$ and $\mathrm{a} / \Lambda \approx 0.2$, and has the available wavelength range corresponds to the value of $\log _{10}(\Lambda /$ $\lambda$ ). The $V_{\text {eff }}$ of this sample is therefore less than 2.405 at all wavelengths. Although single-mode operation will not be defined by multimode at shorter wavelengths), some similar cutoff value $\mathrm{V}_{\text {eff }}$ should be applied. In addition, larger holes make the fiber likely to be multimoded. The gaps between the holes become narrower, isolating the core more strongly from silica in the cladding. Smaller holes make single mode guidance more likely, but the decrease in effective index difference makes the fiber susceptible to bend loss.

\section{Conclusion}

In conclusion, the two dimensional with a triangular-lattice cross sectional pattern of elliptic air holes PCF is investigated in detail by using the PWE method and the dependence of dispersion on structure parameters is calculated. We compared the dispersion relations with various ratios between the circular air holes PCF and the elliptic one. It shows the flatter dispersion curve and single mode in elliptic air holes PCF can be realized by adjusting the width, and the hole pitch. The elliptic air hole PCF can be fabricated by the stack and draw procedure, which will open new possibilities high performance PCF over the conventional fiber.

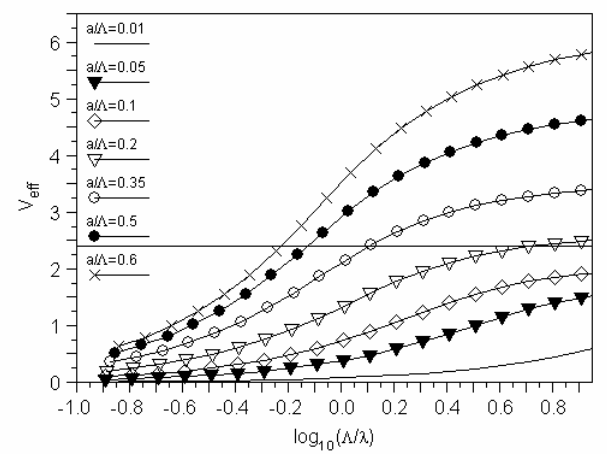

Fig. 5: Variation of $V_{\text {eff }}$ with $\log _{10}(\Lambda / \lambda)$ for various relative ratio $\mathrm{a} / \Lambda$. The horizontal line corresponds to the single mode condition, $\mathrm{V}_{\text {eff }}=2.405$, the cutoff $\mathrm{V}$ value for a step-index fiber.

\section{Acknoledgement}

This project is financially sponsored by National Science Council (grand no. NSC-2112-M-231-001) and Ministry of Economics (grand no. 94-EC-17-A08-S1-0006).

\section{References}

[1] Night J C, Russell P S. Science, 296,276 277(2002).

[2] Night J. C., Birk T. A., Russel P. S. J., Optt. Lett., 21(19), 1547 1549(1996)

[3] Night J C, Broeng J, Birk T. A., Science, 282, 1476 1478 (1998)

[4] Cregan R. F., Mangan B. J., Kright J. C., Science, 285, 1537 1539(1999)

[5] T. M. Monro, P. J. Bennett, N. G. R. Broderick, Opt. Lett., 25(4), 206 208(2000)

[6] T. A. Birks, J. G. Knight, P. S. Russel, Opt. Lett., 22(13),961 963(1997) 
[7] G. B. Ren, Z. Wang, S. Q. Lou, S.S. Jian, Chinese Journal of Laser, V31, 1006(2004).

[8] See http://ab-initio.mit.edu/mpb

[9] Jes Broeng, Optical Fiber Technology 5, 305330 (1999) 